\title{
LA INFLUENCIA DE LA ALQUIMIA MEDIEVAL HISPANA EN LA EUROPA MODERNA
}

\author{
Miguel López Pérez \\ Depto. de Farmacia y Tecnología Farmaceútica \\ Facultad de Farmacia, Universidad Complutense de Madrid
}

\section{RESUMEN}

Los textos pseudolulianos de paracelsistas alemanes en España concretan los años del paracelsismo peninsular. Dichos textos, y algunos más, estaban influenciados por otros anteriores, como los del propio Lulio, Arnau de Vilanova y Juan de Rupescissa, por lo que dichas ideas hicieron un viaje de ida y vuelta y la Alquimia hispana medieval influenció directamente a la europea.

PALABRAS CLAVE: alquimia medieval, España, parcelsismo.

\section{SUMMARY}

The pseudolullian texts of german paracelsists in Spain specify the peninsular paracelsism. This texts, and many others, were influenced by other previous texts, like these of the same Lull, Arnau of Vilanova and John of Rupescissa, that's the reason why this ideas made a return trip and the spanish mediaeval alchemy determined directly to the european alchemy.

KEY WORDS: medieval alchemy, Spain, paracelsism.

\section{LA ALQUIMIA EUROPEA EN LOS SIGLOS MODERNOS}

Las diferencias entre la Alquimia medieval y la moderna suelen atribuirse al efecto Paracelso, a quien se le asigna el hecho que introdujese como tercer componente de la materia la sal, añadiéndolo a los ya existentes mercurio y azufre. Pero hay que reparar en otros hechos que afectaron más o menos directamente, a la evolución de la Alquimia. En primer lugar, el siglo XVI es el siglo de oro de las minas, tanto en Europa como en la Península, gracias, especialmente, a la extracción de metales en América ${ }^{1}$. En Bohemia, los Schonberg explotaron con mucho éxito los yacimientos de Freiburg y los margraves de Branderburgo sacaban oro en Goldenkronach, una

1 Portela, E. (1989), «El beneficio de los minerales de plata en la América Colonial», en PESET, J. L. (Coord.), Estudios sobre la Ciencia, CSIC, Madrid, 1989, v. 10, pp.153-167. 
mina cercana a Bayreuth. En la Península, la llegada de plata y oro de las colonias americanas hizo florecer a toda una serie de especialistas en cuestiones de metalurgia, tales como Alvaro Alonso Barba, Bartolomé de Medina o Bernardo Pérez de Bargas, quien incluso aconsejó en su De re metallica que era bueno conocer los principios alquímicos a todos aquellos que estuviesen relacionados en el sector ${ }^{2}$.

En el lado europeo, George Landmann (Agrícola, 1486-1535), autor de una obra homónima, aunque publicada años antes ${ }^{3}$, contribuyó, como los demás, a la confusión que existió durante el siglo XVI en cuanto a Alquimia se refiere. En su De ortu ${ }^{4}$, cuando trata sobre el origen de los metales, no ayuda en nada a la teoría alquímica sobre la dualidad de los componentes de la materia (azufre, mercurio), ya que dice no ver que existan en las minas metalíferas en todas las ocasiones. Tal motivo le lleva a decir que es un error relacionar los principios alquímicos con los cuerpos ${ }^{5}$ químicos. En el mismo sentido y por las mismas fechas se expresaron otras voces europeas, como la de Vanuncio Biringuccio (1480-1539), un italiano que disertó sobre la naturaleza del fuego en unos términos por los cuales acabó encasillado historiográficamente como químico-metalúrgico. En concreto, aceptó que los minerales fuesen mixtos, pero no que estuvieran compuestos por azufre y mercurio ${ }^{6}$. También lo hizo André Cesalpin (1519-1603), quien, a pesar no creer en los alquimistas, siguió, como ellos, la idea de Aristóteles sobre la generación de los metales, resultado de la condensación de vapores ${ }^{7}$. Pero estos metalúrgicos de profesión pertenecieron a un movimiento más amplio surgido en el siglo XVI. En él se consiguió separar y oponer unos nuevos razonamientos a la tradicional autoridad y, lo más llamativo, la experiencia a la especulación en que estaba sumida la ciencia tradicional, especialmente la médica, centrada en Galeno e Hipócrates. Paracelso aplicó la química a la medicina, Agrícola a la metalurgia y Bernard Palissy (1510-1590) unió química y técnica ${ }^{8}$. Por supuesto, no fueron los únicos. La autoridad de la experiencia también fue proclamada por Leonardo da Vinci (1452-1519), Jerónimo Cardan (1501-1576), Juan Bautista Porta $(1537-1615)^{9} \mathrm{y}$ otros.

2 PÉReZ De BARgas, B. (1568), De re metallica, Madrid, «Prólogo al lector». Edición manejada:. B.N., R-7440.

3 AGRícola, G. (1528), Bermannus sive de re metallica dialogus, Basilea.

4 AgRícola, G.(1543), De ortu et progressu chemiae dissertatio, Basilea.

5 Cuerpo es un término que, en alquimia, designaba al metal.

6 VIRINGUCCIO, V. (1540), Della pyrotechnia libri X, Venecia.

7 Cesalpin, A. (1590), De metallicis lib. III, Roma.

8 Este químico renacentista estudió no sólo los minerales metálicos, sino también la misma tierra en su afanosa búsqueda de nuevos materiales vítreos para la alfarería cuando los europeos ni empiezan a aprender las técnicas persas y ni sueñan con imitar la porcelana china.

9 Nacido en Pavia, inventó la suspensión que lleva su nombre. Como astrólogo, aceptó el ejercicio de la magia natural, razón por la cual Quiroga le incluyó en su Índice de 1583. 
Además, este nacimiento de los especialistas durante el Renacimiento fue acompañado de un crecimiento de personas interesadas en la Alquimia. Algunos magnates, como hemos visto, se procuraban expertos en el ars chymica, querían aprender ellos mismos también qué era eso de la ciencia alquímica. A la vez, este hecho se vio acompañado de otros que ayudaron a esta confusión y crecimiento de seguidores. El tipo de saber de los alquimistas medievales puede ser encuadrado dentro del conocido como artesanal, y, como tal, su transmisión era, eminentemente, por vía oral. La llegada de la imprenta no supuso un inmediato auge de ediciones de textos alquímicos; algo que se alcanzaría durante las primeras décadas del siglo XVI. La inclusión como temática digna de ser llevada a la imprenta no vino per se, sino por medio de la confusión y la aparición de tratados de otro tipo, que sí favorecieron la llegada. Hasta tal punto esto fue así que llega a ser bastante difícil distinguir entre un recetario técnico, una obra de magia y una de Alquimia. Esto fue debido al afán de coleccionar recetas y presentarlas unidas. Estas interferencias, sin embargo, no eran nuevas, ya que la tradición manuscrita ya inició que éstas se multiplicasen. La imprenta, en todo caso, aceleró y potenció aún mas la confusión.

A nivel ideológico, el clima intelectual también contribuyó a esta amalgama. Como decía van Lennep, durante el siglo XVI se mezclaron los extremos y la ciencia simpatizaba con la locura ${ }^{10}$. Podemos ejemplificar esta afirmación si leemos las ideas expresadas por Pico della Mirandola (1463-1494) o Marsilio Ficino, dos hermetistas y cabalistas de primer orden muy conocidos en la Península ${ }^{11}$. Por ejemplo, Pedro Ciruelo se carteaba con amigos de Oxford sobre estos temas, estuvo muy influenciado por d'Etaples (1455-1537), un lulista acérrimo, y dialogó con el mismo Pico. Los canónigos adscritos a la Catedral de Burgo de Osma conocieron perfectamente las ideas de Marsilio sobre el hermetismo ${ }^{12}$, lo mismo que ocurriera con los de la catedral de Toledo ${ }^{13}$, o en el Monasterio de San Lorenzo de El Escorial ${ }^{14}$.

La Alquimia pasó a ser algo semejante a una gran doctrina iluminista para los católicos en un momento en que abundaron las herejías y cuando las doctrinas teosóficas y mágicas se extendieron por Europa, durante los siglos XIV y XV. Llegado el Renacimiento, la Alquimia quedó unida a la cábala, a la magia y a la teosofía. Pero hubo de compartir escenario con, al menos, otros cinco compañeros, como el neoplatonismo de Marsilio Ficino, el neo-pitagorismo de Nicolás de Cusa, el cabalismo

\footnotetext{
10 LENNEP, J. van (1978), Arte y Alquimia, Editora Nacional, Madrid.

11 Suquía Goicoechea, A. (1988), Un maestro y un alumno de Alcalá, Pedro Sánchez Ciruelo e Íñigo de Loyola, R.A.H., Madrid.

12 Poseían un ejemplar de Ficino titulado Comentarium in librum Trismegisti, del siglo XV. Biblioteca Catedralicia, códice 25 y A.H.N., microfilm 7144-45.

13 Ficino, M. (1561), Marsilii Ficini opera duobus voluminibus, Basilea.

14 FICINO, M. (1524), Platonica theologica, Venecia, 1524, ESC., 73-IV-1. FICINO, M. (1576), De vita et Mercurius Trismegistus, Basilea, ESC., 18-IV-1 y 2.
} 
cristiano de Reuchlin y Pico y, en la Península, el lulismo hermético. En el Humanismo, el celo por una pulcritud intelectual llevó a los protagonistas no a aislar otras doctrinas, sino a darse de cara con una multitud de ellas, con concepciones de las cosas distintas, y, por supuesto, a relacionarlas. La teosofía y todas las demás formas de pensamiento hubieron de convivir en el Renacimiento de forma descarada, sus identidades se mezclaron, formando una amalgama donde es difícil discernir cuál de ellas es la predominante, aunque no por ello hemos de pensar que su estudio resulta inaccesible, sobre todo si entendemos que, en realidad, todo eran diferentes puntos de vista de la filosofía de la naturaleza. La ciencia mística, por otra parte, contenía en sí el germen de una especulación creadora. Lo ocurrido fue el nacimiento y florecimiento de esta semilla. Por mucho que los historiadores de la ciencia pretendamos realizar un estudio aséptico de la ciencia moderna hasta la llamada Revolución Científica, o aún más tarde, ya que las raíces herméticas de muchos de sus adalides son patentes hasta entonces, nunca veremos el modo en que pudo revolverse contra sí y desterrar sus fantasmas de ocultismo. Esta relación ciencia-magia, o cienciahermetismo ha sido vista como una racionalidad irracional por algunos filósofos ${ }^{15} \mathrm{e}$ historiadores. De hecho, la ciencia realizada en el periodo que comprende este trabajo, no pudo deshacerse de su lastre mágico.

Este ejercicio vio su culminación a principios del siglo XVII, y ocupará prácticamente todo su primer tercio. Se conoce como un movimiento intelectual llamado Pansofia, y abogaba por un conocimiento total y universal. Sus protagonistas más destacados fueron Comenius (1592-1670) ${ }^{16}$, Heinrich Kuhnrath (1555-1605) $)^{17}$ y los rosacruces. El propio nacimiento de la semilla llevaba el final de la ciencia mística y la posibilidad de la libre especulación intelectual que traía consigo fue desarrollada al máximo. Visto con sus propios ojos, la amplitud de los presupuestos metafísicos en los que se basó la filosofía natural del siglo XVI, como el hermetismo y el neoplatonismo, hizo posible que los especuladores surgidos al amparo de tal paisaje intelec-

15 Hesse, M. B. \& Rossi P. (1988), Los filósofos y las máquinas (1400-1700), Labor, Madrid.

16 Jan Amos Komensky (Comenius) nació un 18 de marzo en Moravia. Fue rosacruz y alquimista, además de discípulo de Johan Valentin Andreae. Ingresó de joven en la Iglesia de los Hermanos Moravos (concretamente en al del maestro Jan Hus). Huérfano desde joven, no pudo estudiar hasta los 16 años. Al mostrar sus dotes fue enviado por la Unidad de los Hermanos (moravos) a la Universidad de Herborn (Nassau), donde estudió Teología y lenguas vivas. Luego ejerció de pastor en Fulnek (Moravia). Al inicio de la guerra de los 30 años su casa fue asaltada y su esposa e hijos muertos. Vagó por los pueblos de toda Europa, fue echado de Bohemia y se refugió en Leszno (Polonia), donde la Unidad de Hermanos tenía un colegio famoso. Fue protegido por Luis de Geor, su mecenas sueco, pero tropezó con los luteranos de Estocolmo. El 25 de abril de 1656 se refugió en Nardem (Holanda), donde murió el 15 de noviembre de 1670. En el movimiento pansofista, quiso establecer una metodología conciliadora entre las diversas corrientes religiosas, las ciencias y las técnicas. Para él, todos lo conocimientos tienen una única fuente que anima al Todo en todas sus partes, algo semejante a un esbozo de sinarquía. BLEKASTAD, M. (1969), Comenius, Oslo.

17 KHUnRATH, H. (1602), Amphitheatrum sapientiae aeternae solius verae christiano-kabalisticum, divino-magicum nec non physico-chymicum tertriunum catholicon, Hanovide, Guilielmus Antonius. 
tual pudiesen, incluso, obviarlo y, teniendo como herramienta el uso de dicha libertad, además, transformaron dicha especulación amplia en una experimentación concreta. En ella, ya no hubo que justificar el a priori tras la experiencia ya que los fines últimos eran más moderados, tales como la mejora de un medicamento, una forma de destilar más efectiva, la mejor curación terapéutica de una enfermedad, etc. quedando lejos la posibilidad de la Piedra Filosofal, la medicina universal o cosas semejantes.

Volviendo al hilo conductor, no está determinado con exactitud qué grado de influencia ejerció el movimiento pansofista en la mitad del siglo XVII, pero lo podemos intuir gracias a cómo estaban las cosas en el último tercio y cómo después. Debió ser una época burbujeante, llena de actividad y de, claro está, experimentación. La historiografía de la ciencia se ha aplicado bastante al estudio de estos años, y aún sigue haciéndolo. Sea lo que fuere que ocurriese, y sobre todo con estos intentos de amalgamar todos los conocimientos, la Alquimia salió transformada en Europa. Glauber inició el acercamiento, aunque muy lentamente, hacia la química. Por otro lado, los que se consideran adalides de la Revolución Científica, como Boyle, Newton, Leibniz, y otros, seguían creyendo en la transmutación. Y, una tercera vía, la que siempre está paralela y inmutable a los sucesos, la de los alquimistas tradicionales y tradicionalistas, seguía su camino diferenciado a manos de Juan Federico Helvetius $(1625-1700)^{18}$, Pierre Borel $(1620-1671)^{19}$ y Elías Ashmole $(1617-1692)^{20}$. Los matices a las teorías de los alquimistas, semejantes a los habidos con Palissy y otros ya mencionados antes, se repitieron en esta «edad oscura» de mediados del siglo XVII y hasta su final.

El ambiente reformador de las ciencias del siglo anterior se continuó, destacando Galileo, Descartes, Boyle y Bacon. Ya el tono opositor se aleja del enfrentamiento, ahora es cuando realmente se da el cambio de la autoridad tradicional y la especulación por la experiencia y la razón. Por fin la ciencia perdía ese lastre que la anquilosaba. No obstante existió cierta resistencia a esto durante la primera mitad de siglo, debido, principalmente a la guerra de los 30 años, a los agitados años del reinado de Luis XIII y la minoría de Luis XIV. Muchos de los que opinaron sobre la Alquimia tienen algo en común: estaban todos en la misma arena y sus concepciones mágicomísticas sobre un universo inter-relacionado mediante unas fuerzas no siempre comprensibles, visibles y capaces de ser dominadas son notables, no pasan desapercibidas. De ellos un buen ejemplo fue Juan Bautista van Helmont (1577-1644). No publicó nada en vida y sus textos los reunió su hijo, François Mercurio van Helmont,

\footnotetext{
18 Helvetius, J. F. (1650), De alchymia apuscula complura veterum philosophorum, Frankfurt.

19 BoRel, P. (comp) (1654), Bibliotheca chemica seu catalogus librorum philosophorum hermeticorum, París.

20 Ashmole, E. (ed.) (1650), Fasciculus chemiaica abstruae hermeticae scientiae ingressum progressum corinden explicans, Londres.
} 
bajo el título Ortus Medicinae ${ }^{21}$. Laín Entralgo le definió como iatroquímico y sus ideas sobre la Alquimia eran tan similares a la de los maestros medievales, que sólo se diferencian en que no cree que todo venga de algo universal, dada la variedad que presenta la naturaleza, a pesar de admitir como principios al mercurio, azufre y sal. Algo similar pensó el citado Robert Boyle (1627-1691), nacido el mismo año de la muerte de Francis Bacon. Acepta la división elemental de la naturaleza, pero no admitió que el agua fuese uno de ellos. Otro revisionista, tan sutil como los anteriores fue Kunckel (1630-1702), personaje que acepta el método experimental a la vez que a la Alquimia ${ }^{22}$. Los matices, por muy nimios que nos parezcan, las opiniones divergentes, aunque sean en lo más mínimo, los detalles ofrecidos para explicar algún concepto y sus pequeñas diferencias entre ellos son nuestra mejor herramienta para poder ver cómo las ideas y las posiciones no alcanzaron una estabilidad duradera, para ver cómo se fueron transformando en un proceso de larga duración, en parte acumulativo y en parte no. En realidad, fueron la suma de dichas inestabilidades la que engendró otras, estas sí más sólidas, que provocaron un efecto contrario a su naturaleza: volvieron móviles posiciones bien asentadas. Un claro ejemplo del siglo XVI fue el del médico Daniel Sennert (1572-1637)23. Vivió en las décadas de mayor actividad en la reciente relación Alquimia-Terapéutica-Sanidad. Criticó a Paracelso y sus sectatores, por un lado, ya que no creía en la medicina universal o en el alkhaest. Pero, por otro, también se situó en contra de los galenistas por «resistirse al progreso de la Medicina», y por su obstinado talante conservador ${ }^{24}$.

Toda esta actividad de pensamiento especulativo tuvo su lado público y su grado de corporativismo. El germen de las sociétés savantes está, nada más y nada menos que en la Academie des Secrets, una sociedad fundada por Jean Baptista Porta (1540$1615)^{25}$, unos de los doctores más renombrados de magia natural, hecho que ocurrió

21 Helmont, J.-B. van (1648), Ortus medicinae: id est initia physicae inaudita progressus medicinae nouus in morborum ultionem ad vitam longam, Daniel Elzewir, Amsterdam.

22 Johan Kunckel von Lowënstein podría considerarse un químico progresista lento, encasillado en el campo mineral y orientado paulatinamente a la química. En 1679 inventó el cristal de rubí auténtico. Comenzó su carrera en Bohemia trabajando para los Saxe-Lauenburg. GANZEMÜLLER, W. (1956), Beiträge zur Geschichte der Technologie und der Alchemie, Weinheim, pp. 46-51, 105-115 y 192-203; PARTINGTON, J. R. (1961), A History of Chemistry, Londres, II, pp. 361-377.

23 Daniel Sennert fue un famoso médico de Brislau, profesor de Medicina en Wittemberg. Sennert, D., (1629), Medicina practica, Lugduni; (1637) Physica_hypomendata: de rerum naturalium principiis, Lugduni; (1665a) Instituonionum medicinae, Lugduni; (1665b) Epitome universam D. Sennerti doctrina, Colonia y (1665c) Opera omnia medica, Wittemberg. Thorndike, L. (1946), History of Magic \& Experimental Science, Nueva York, VII, cap. 7.

24 SENNERT, D. (1665d), De chemicorum cum aristotelicis et galenicis consensu et dissensu, Wittemberg, «Pars prima», p. 12.

$25 \mathrm{Su}$ Magia naturalis fue traducida a todas las lenguas de Europa. 
en su propia casa ${ }^{26}$. Otro grupo, teniendo por cabeza principal al médico bávaro Michel Ferh, y acompañado de G. B. Metzger, G. B. Wolfarth y más médicos alemanes iniciaron en 1651 la andadura de la Academie des curieux des sciences, donde se estudiaba conjuntamente tanto Medicina, como Química e Historia. Ya a fines de siglo, la Academia de Fisiócratas de Siena, nacida bajo el patronazgo de Francisco de Médicis en 1691 tuvo entre sus miembros muchos que experimentaron la Alquimia, a pesar de adoptar el método experimental. Fue en estos años de la segunda mitad del siglo XVII cuando ya podemos hablar con menos intranquilidad de la existencia de la química farmacéutica.

Ya en el siglo XVIII la Alquimia adosada a la Terapéutica y a la Sanidad se transformó, previa disolución, en la química cercana a la que nos ha llegado, gracias a Lavoisier y a los que sirvieron de puente, como Stahl y la teoría del flogisto. Para Lavoisier, el cuerpo era una noción incompatible con la transmutación. Pero aún veremos que siguen los alquimistas tradicionales, representados por Pernety o Lenglet du Fresnoy. Además, la unión que hubo en el Renacimiento con la teosofía volverá a surgir entre los místicos alemanes, especialmente con Karl von Eckartshausen (1752-1803) ${ }^{27}$. En 1732, Boerhaave (1668-1738), en su Historia de la Química dividió a los primeros químicos importantes en cuatro clases: Los escritores «sistemáticos» que reducían las operaciones de la química a la forma de sistemas, especialmente para la preparación de remedios químicos, como fueron los que se alejaron de Paracelso, incluidos muchos espagiristas, los «químicos-metalúrgicos», como Agrícola, los escritores alquimistas, como Paracelso y Sendivogius y los perfeccionadores químicos de la filosofía natural, como Robert Boyle (1627-1691).

Si bien es una clasificación muy simplista, en ella se contienen casi todos los tipos de personas que se acercaron a la Alquimia, pero sin diferenciaciones internas. Además, encontramos protagonistas que pertenecieron a más de una clase, como veremos en su lugar.

\section{LOS PRIMEROS ACERCAMIENTOS ENTRE ALQUIMIA Y TERAPÉUTICA}

Si nuestra intención fuese rastrear las ideas entre cada uno de ellos, ver su influencia, analizar sus relaciones y contactos, y hacerlo en un encadenamiento, se podría establecer una secuencia desde los platónicos hasta Nicolás Lémery, desde un alquimista a otro, desde la Edad Media hasta el siglo XVIII, y así sin interrupción.

\footnotetext{
26 Si bien la historiografía ha otorgado a esta academia el título de la primera, no podemos olvidar que ya estaba fundada la Accademia Segreta de Girolamo Ruscelli, más conocido como Alexo Piamontés. EAMON, W. \& PAHEAU F. (1984), «The Accademia Segreta of Girolamo Ruscelli. A SixteenthCentury Italian Scientific Society», Isis, 75, 327-342.

27 ECKARTSHAUSEN, K. von (1801), Khemische Versuche, Ratisbona, Montag und Weiss.
} 
Podríamos ir de Alberto Magno a Tomás de Aquino, a Llull, a Giorgi, a Fludd, a Maïer, a Tritemio, a Paracelso, a Böhme, a Gichtel, a Schott, a van Helmont y a Leibniz «saltando» de uno a otro sobre las mismas ideas.

Con todo lo dicho, sin embargo, hay que buscar en la Edad Media para rastrear los orígenes del desarrollo de la Alquimia moderna europea. Si pudiésemos dejar todas las piezas en una mesa y verlas desde la distancia, notaremos algo realmente curioso sobre lo ocurrido en la Península. De ella salieron las ideas que se implantaron en toda Europa. Los textos más renombrados de Alquimia se atribuyen, durante toda la Edad Moderna a los clásicos, como Arnau de Vilanova, Rupescissa o Lulio, todos ellos peninsulares. Estos autores son nombrados una y otra vez como los verdaderos filósofos, junto a otros, no menos conocidos, como Tomás de Aquino, por ejemplo. El estudio del origen de estas influencias en la Medicina química de los siglos XVI y XVII se hace imprescindible para intentar comprender en qué estado llegó la Alquimia a iniciar su relación en el periodo que nos ocupa para este trabajo.

Por otra parte, es evidente que el uso de metales y minerales en la Terapéutica estaba ya muy extendido ya antes de Paracelso. Incluso también lo estaba el uso del mercurio, del antimonio y de los ácidos minerales en la Medicina interna ${ }^{28}$, aunque estaban más generalizado como remedios externos. La novedad, que tampoco resulta serlo tanto, es que el número y cantidad de los preparados químicos de aplicación interna superarán en número a los externos. Además, segunda novedad, estos medicamentos internos tienen su propia forma de elaboración, unos métodos escasamente usados con anterioridad; y son los propuestos por la Alquimia: destilación, solución, etc. En realidad los medicamentos químicos reciben su nombre no del origen de sus componentes, sino del método de preparación, cuyas referencias nos llevan a nuestros chymicos. Estos problemas sobre la preparación de remedios terapéuticos viene por los diferentes concepciones entre los médicos, especialmente los galenistas, y los chymicos. Los primeros realizaban un compuesto de las cualidades, sumando las de los componentes de dicho preparado (cualidad fría más cualidad seca, por ejemplo). En cambio, los chymicos extraían las de cualquier mixto y las purificaban tras eliminar las heterogeneidades impuras, por lo que decían que las exaltaban todas juntas, en forma de esencia. Esta esencia podría, también, tener las cuatro cualidades (fría, seca, húmeda y caliente), estado en que recibía el nombre de quinta esencia.

A mediados del siglo XIV aparecen tratados con una clara diferencia de los existentes un siglo antes. Estos últimos, como los de Michael Scot o Geber nos ofrecen un repertorio de procesos bastante amplio, nítido e inteligible. Michael Scot ha sido calificado en muchas ocasiones como el primer alquimista europeo ${ }^{29}$ y ya fue men-

28 Multhauf, R. P. (1954), «John of Rupescissa and the Origin of Medical chemistry», Isis, 45, pp. 359-367.

29 Michael Scot («el escocés») ( $i$-1235) estuvo en la escuela judía de Toledo hacia el año 1217 traduciendo textos árabes. En el año 1220 ejercía medicina en Bolonia, los papas Honorio III y Gregorio IX 
cionado por Dante (1265-1321) como mago en su Divina Comedia ${ }^{30}$. Otra figura relevante de la alquimia medieval fue Geber, o Djabir ben Hayyan (i-815), seguidor de Aristóteles y versado en matemáticas, medicina, astrología y música. Como otros contemporáneos, Geber se dedicó a la investigación científica, actividad que prescribe explícitamente el Corán. Entre otras cosas, describió la cristalización como medio para purificar los preparados químicos, varios métodos para obtener el ácido sulfúrico, el ácido nítrico, el nitrato de plata, el cloruro amónico, el arsénico y el vitriolo de hierro. En la parte alquímica, sus investigaciones le llevaron a realizar aleaciones (de mercurio con oro, plata, plomo, estaño y cobre), óxidos y enlaces entre metales (principalmente mercurio) y azufre. Pero sus métodos destacan por sentar las bases de la química experimental ya que observaba el desarrollo de las reacciones químicas en unas condiciones establecidas artificialmente, variando de forma arbitraria los parámetros y siguiendo los efectos que dichas modificaciones generaban. $\mathrm{O}$ sea, en lenguaje alquímico, procesos de sublimación, calcinación, destilación, congelación y otros ${ }^{31}$.

Algunos historiadores de la ciencia medieval han logrado elevarse por encima de Geber y, una vez tratado con detenimiento, han reflexionado sobre la alquimia desarrollada en el mundo islámico y sobre el tipo de ciencia que se practicaba. Es el caso

\footnotetext{
le distinguieron con beneficios eclesiásticos. Estuvo en Sicilia al servicio de Federico II Hohenstaufen. Tradujo al latín obras de Aristóteles, Averroes y Avicena. Estudió astronomía, cálculo del tiempo, astrología, medicina y cosmología. Consideraba, como Avicena, a la materia dividida en los tres principios alquímicos (sal, mercurio y azufre). Una excelente biografía ya fue hecha en el siglo XIX por John Wood Brown, imprescindible para entender a Scot y su relación con la alquimia, aunque los planteamientos metodológicos del autor no son los óptimos. WOOD BROWN, J. (1897), An inquiry into the life and legend of Michel Scot, D. Douglas, Edimburgo; SINGER, D.W. (1930), «Michael Scot and Alchemy», en Isis, 13, pp. 5-15; THORNDIKE, L. (1965), Michael Scot, Londres; HANSKINS, T. L. (1928), «The <Alchemy> adscribed to Michael Scot», en Isis, 10, pp. 350-359; THOMPSON, S. H.(1938), «The text of Michael Scot's Ars Alchemie», en Osiris, 5, pp. 523-559.

30 Dante Alighieri (h. 1300), La divina comedia, Inferno, canto XX, versos 115-117: «Aquél otro de allí, menudo y flaco, era Michael Scot, que ciertamente los trucos de la magia conocía». Edición manejada: (1997), Alba, Madrid, p. 97.

31 SezGin, F. (1964), «Das problem der Gabir ibn Hayyan im lichte neu gefunener Handschriften», en Zeitschrift der eitschen Morgenländischen Gesdellschaft, 114, pp. 255-268; PLESSNER, M. (1965), «Gabir ibn Hayyan und die Zeit der Enstsehung der Arabischen Gabir-Schriften», en Zeitschrift der eitschen Morgenländischen Gesdellschaft, 115, pp. 25-26, PLESSNER, M. (1969), «Geber und Jâbir ibn Hayyan: An Authentic $16^{\text {th }}$ quotation from Jâbir», en Ambix, 16, pp. 113-118. Pseudo-Geber (1682), Gebri regis Arabum philosophi perspicacissimi Summa Perfectionis magisterii in sua natura, Brunonen Taken, Dantkig; BERTHELOT, M. (1898), La chimie au moyen âge, Imprimerie Nationale, París, pp. 336350; DARMSTAEDTER, E. (1922), Die Alchimie des Geber, Berlín; DARMSTAEDTER, E. (1924), «GeberHandschriften: Vorläufige Metteilung», en Chemiker Zeitung, 48, pp. 441-442; DARMSTAEDTER, E.(1925), «Liber misericordiae Geber. Eine lateinischen übersetzung des grösseren kitab alrahma», en Archiv für Geschichte der Medizin, 17, pp. 181-197; CORBIN, H. (1950), Le livre des glorieux de Geber, París, Eranos Jahrbuch; HOLMYARD, E. J. (1958), The works of Geber, englished by Richard Russel, 1678, Londres, J. M. Dent \& Sons; JorgenSEN, B. J. (1958), Testamentum Gebri, Londres, J. M. Dent \& Sons.
} 
de Paul Kreaus, quien nunca se convenció de la originalidad de su alquimia, aunque tampoco afirmó que fuese una simple copia de los postulados griegos, ni que la alquimia el Islam muriese como un traspaso a Occidente sin más ${ }^{32}$. No obstante, hay que agradecerle que demostrase la existencia de pseudografía geberiana incluso dentro del Islam.

\section{LOS TEXTOS Y LOS AUTORES QUE INFLUYERON EN LA ALQUIMIA EUROPEA.}

Pero, como se dice arriba, un siglo después, a mediados del XIV, los textos parecen centrarse en la descripción minuciosa de tan sólo dos cosas: la destilación y la disolución, todo ello combinado con el trabajo sobre los ácidos minerales. De dichos textos destacan varios, como la Practica vera alchimia de Hortulanus, el Rosarius Minor, el Testamento, falsamente atribuido a Lulio (1235-1315), los textos de Arnau de Vilanova (1245-1313) y el Liber Lucis de Juan de Rupescissa (1328-1385). Resulta, cuanto menos curioso, que estos escritos aún fuesen leídos a fines del siglo XVI. Así, el Liber Lucis llegaría hasta la edición de Manget de 170233; el Testamento de Pseudo-Lulio, en manuscrito, y aparte de las múltiples ediciones europeas de las que luego hablaremos, estaba en El Escorial desde finales del siglo $\mathrm{XVI}^{34}$ y el Rosarius Minor, también fue manuscrito en la Península en la mismas fechas, concretamente en $1596^{35}$.

En todos ellos, encontramos en común, aparte de las descripciones sobre los trabajos de destilación, que ya aparece muy bien asentada en la Alquimia cristiana bajomedieval la concepción de los elementos mercurio y azufre como componentes de toda materia, y que la Piedra Filosofal se realiza en siete pasos, además de los conceptos del oro potable, la quinta esencia, trabajos sobre el mercurio sublimado, tan en boga durante los siglos XVI y XVII, los sublimados corrosivos, etc.

La historiografía peninsular ya prestó atención a los tres alquimistas españoles, llegándolos a calificar como el triunvirato de la Ciencia catalana del siglo XIV, palabras que recogiera Multhauf en el artículo ya mencionado ${ }^{36}$. Pero lo que aquí nos interesa son dos cosas: por un lado, establecer la influencia que dichos autores se ejercieron entre sí y, por otro, averiguar y establecer, en su caso, la influencia que ejercieron en Europa. Esto último es tremendamente importante, ya que en caso de

32 KREAUS, P. (1942-1943), «Jâbir ibn Hayyan. Contribution a l'histoire des idées scientifiques», en Memorias presentadas al Instituto egipcio de El Cairo, I, pp. 102-117.

33 MANGET (1702), Bibliotheca chemica curiosa, II, 84-86.

34 PSEUdo-Lulio, R. (s. XVI), Raymundi Lulii maioricani philosophiae codicilus, sive testamentum quod eiusdem theorica dicitur, s.1.

35 Anónimo (s.f.), Rosarius minor non imperitii de cognitione rerum metallorum, s.1.

36 Menéndez y Pelayo, M. (1880), Historia de los Heterodoxos españoles, Madrid, Librería Católica de San José, I, p. 500. 
resultar positivo, podremos afirmar que la Alquimia moderna debe su base, o gran parte de ella, a estos autores peninsulares. Si ello fuese así, estaríamos hablando, en una perspectiva que abarcase hasta el final del siglo XVII, de unas ideas que viajaron en un recorrido de ida y vuelta, ya que, como veremos, volvieron a entrar aquí, aunque no de la misma forma que salieron, sino generando la presencia de la ideas de Paracelso, ya incrustadas en dichos textos ${ }^{37}$. Es decir: la Alquimia peninsular orientó a la europea, ésta la asumió, digirió y transformó con sus aportaciones originales y propias, pero siempre basadas en las auténticas. Una vez así, volvieron a penetrar en la Península a través de los textos.

La idea de la influencia de Rupescissa en los textos pseudográficos atribuidos a Lulio, y de forma grave y contundente, no ha sido considerada por la Historia de la Ciencia como un elemento a tener en cuenta. De lo que estamos seguros es que dicha influencia aparece en los textos pseudográficos de Lulio desde el siglo XIV, donde se siguió en varias ocasiones las indicaciones de Rupescissa, llegando, incluso a poner sus palabras de ejemplo a seguir y demostración de las suyas propias:

Por esa razón los filosofos antiguos como Hermes, Sócrates, Platón, Aristóteles decían que no morían y que tenían en estima sus cuerpos porque eran indestructibles, y ello es cierto hablando de forma natural, si el Altísimo no hubiera constituido limites, etc. Dejando a un lado las opiniones de éstos, descendamos a la materia de la que queremos tratar y veamos lo que dice el hermano Johannes Rupescissa, de la orden de los frailes menores, el cual escribió que poseía la ciencia, el espíritu profético y habló así: Toma el vino, noble, alegre, joven, lleno de sabor, el mejor que puedas encontrar. Y esto lo interpretan los ignorantes y los no iniciados al pie de la letra; comienzan la obra y al final no descubren nada ${ }^{38}$.

Por supuesto, el anacronismo evidente nos hace rechazar cualquier idea sobre la realidad de dichos flujos de pensamiento. Rupescissa vivió en la generación posterior a la de Arnau y Lulio. Por otra parte, la influencia los dos últimos está sobradamente demostrada, ambos pudieron encontrar se muchas ocasiones, tanto en Barcelona en 1286, año en que residía allí el primero, como en alguno de los catorce viajes que Lulio hiciera a Italia, o en la Universidad de Montpellier, donde Arnau llegó a ser doctor en Medicina.

Pero analicemos la misma cuestión proyectada en la Medicina química de los siglos XVI y XVII. Uno de los trabajos más conocidos de Juan de Rupescissa es su De consideratione quinta essentiae ${ }^{39}$, texto que aparecería también atribuido a Lulio por

37 Sobre el paracelsismo en la Península en estas fechas es imprescindible consultar los trabajos de LóPeZ PiÑERO, J. M. (1973), «Paracelso and his Work in $16^{\text {th }}$ and $17^{\text {th }}$ Century Spain», Clio Medica, 8 , pp. 113-141; y «El paracelsismo en la España del siglo XVI», en el estudio introductorio a Santiago, D. de (1994), Arte separatoria, Alicante, Instituto de Cultura «Juan Gil-Albert», pp. 11-30.

38 PSEUdo-Lulio (s. XIV), Da comienzo la epístola referente al oro potable y a la Piedra Filosofal enviada al sumo pontifice, s.l.

39 RUPESCISSA, J. (s. XV), Liber de consideratione quinta essentiae, s.1. 
las mismas fechas ${ }^{40}$. El caso contrario, la influencia de Lulio en Rupescissa sí parece más probable, aunque seguimos dudando de que vayan más allá de las cuestiones alquímicas y las de Filosofía de la Naturaleza ${ }^{41}$. Aquí nos guiaremos por las opiniones vertidas en su día por Lynn Thorndike ${ }^{42}$ y por Robert P. Multhauf ${ }^{43}$, además de los estudios recientes de la profesora de la Universidad de Siena, Michela Pereira, sobre la expansión del paracelsismo en Italia a través, especialmente, de las diferentes ediciones de los textos pseudográficos lulianos ${ }^{44}$.

Sobre la consideración de la quinta esencia se inicia con un alegato sobre las excelentes virtudes terapéuticas del alcohol y luego, de la quinta esencia. Ésta sería un producto elaborado cuya principal característica sería la incorruptibilidad y otorga al vino destilado la consideración de llevar impreso el sello de los cuatro elementos. Es por ello que nos dio a conocer varios métodos de destilación del vino. A continuación dice que dicho producto es necesario para la elaboración del oro potable, que no es sino un medicamento. No obstante no deja cerrada la posibilidad de obtener la quinta esencia a partir de otros componentes. De todas formas la utilidad que se le aplica es la de mezclarse con el oro y disolverlo. Estas ideas resultan ser muy importantes si queremos compararlas con las que en el siglo XVI se tuvieron sobre la elaboración del oro potable y los atributos dados a la quinta esencia, cuestión que no dejaría de estar en primera línea de la atención de la Terapéutica hasta fines del siglo XVII.

Otro producto que Rupescissa atribuye cualidades quintaesenciales es el mercurio sublimado, otro disolvente del oro. Debemos pensar que se podía llamar disolvente o menstruos, además de los ácidos corrosivos, ya conocidos, al espíritu de vino rectificado y al mercurio sublimado, ya que, como poco, alteraban el estado físico aparente del oro. Es en este punto donde podemos pensar que aún no existe distinción entre la Alquimia metalúrgica y la medicinal o terapéutica. Como se hiciera también en los siglos modernos, el mercurio era sublimado por nuestro autor con sal y vitriolo. El método será copiado de forma idéntica por los médico-químicos más tarde y por muchos otros alquimistas, aunque algunos, como veremos, usan la sal amoníaca, no especificado por Rupescissa. De cualquier forma, sus conceptos sobre la quinta esencia

40 Pseudo-Lulio (s. XIV), De consideratione quinta essentiae libri duo, s.1.

41 Rupescissa, J. (1561), Consideratione quintae essentiae rerum omnium () Raymundi Lulij ars operativa, Basilea.

42 ThORNDIKE, L. (1946), III, 725-734: «Manuscripts of John of Rupescissa. <De consideratione quinta essentiae $>$. Extracta from... latin text...»

43 Multhauf, R. P. (1966), The Origins of Chemistry, Oldbourne, Londres, 1966, cap. 10, pp. 201236: «Medical chemistry».

44 CRisciani, C. \& PEREIRA, M. (1996), L'arte del sole e della luna. Alchimia e filosofia nel Medioevo, CISAM, Spoleto. PEREIRA, M., «L'elixir alchemico fra artificio e natura», en NEGROTTI, M. (ed.) (1955), Artificialia. La dimensioe artificiale della natura umana, Bolonia, CLUEB, pp. 255-267. PEREIRA, M. (1992), «Un tesoro inestimable: elixir e prolongatio vitae nell'alchimia del'300», en Micrologus. Natura, scienze e società medievali, 1, pp. 161-187. 
serán vueltos a ver, ya sea en la preparación del mercurio, sublimado o no, en la del oro potable y en la de otros preparados químicos o médico-químicos a lo largo de la Edad Moderna en muchos textos europeos. En especial, me refiero a varios en concreto, como los de Felipe Ulstadio de Nüremberg ${ }^{45}$ o los de Paracelso ${ }^{46}$. Incluso se pueden rastrear dichas ideas en el metalúrgico Jerónimo Brunswick (1450-1533) ${ }^{47}$, quien reparó en Rupescissa de forma explícita para la aclaración de algunos conceptos.

Esta transmisión de ideas sobre los conceptos de quinta esencia originales de Rupescissa en Europa, ya sea directa o indirectamente, es decir, nombrándole o no, no era ajena en la Península, especialmente en cuanto al conocimiento de los textos de los dos primeros autores, Ulstad y Paracelso, se refiere. Respecto del primero, senador en la ciudad de Nüremberg a fines del siglo XV, contamos con ejemplares de su Coelum philosophorum desde muy temprana fecha. Así, podemos ver las tres primeras ediciones que hiciera de este texto Johann Grunyngen, entre 1525, año de la primera, y $1528^{48}$, todas ellas en Estrasburgo, además de otra, bastante curiosa, también de 1525 , pero de Friburgo ${ }^{49}$. Aún más, una edición posterior, ya del año 1535 nos dice que los secretos de la naturaleza que se encuentran reunidos en dicho texto, pertenecen a textos de los principales protagonistas de este apartado, tales como el propio Ruspescissa, Lulio y Arnau de Vilanova ${ }^{50}$. En el Prefacio al Coelum, Ulstad dijo que, en su tiempo, los textos de Lulio estaban en muchas manos, o, dicho con sus palabras: «Ad paucorum enim manus pervenisse video ${ }^{51}$.

Lo mismo nos ocurre con las Archidoxias de Paracelso, aunque las ediciones sean posteriores; en concreto contamos con las de Frankfurt de los años $1584^{52}$ y $1603^{53}$. No obstante, también tenemos, aunque tardía, y seguramente ampliada, la que hiciera Francesco Collona (1433-1527), un dominico veneciano, hermetista, profesor de

\footnotetext{
45 Ulstad, Ph. (1925a), Coelum philosophorum, Friburgo.

46 PARACELSO (1925), Archidoxorum libri, Basilea.

47 BRUNSWIK, H. (1500), Liber de arte distillandi compositis, Johan Gruningen, Estrasburgo.

48 UlSTAD, Ph. (1925b), Coelum philosophorum, seu de secretis naturae, Johan Gruningen, Argentorati, (1526), Coelum philosophorum, seu de secretis naturae, J. Gruningen, Argentorati.

49 UlSTAD, Ph. (1525c), Coelum philosophorum, seu de secretis naturae, Friburgo, 1525.

50 UlstAD, Ph. (1535), Coelum philosophorum, seu de secretis naturae liber.../ ex authoribus... Iohanne de Rupescissa, Raymundo Lullio, Arnoldo de Vilanova, Albertoque Magno, a Philippo Ulstadio... collectus, Iacobus Cammerlander, Argentorati.

51 Sobre Ulstad: GMELIN, J. F. (1797-98), Geschichte der Chemie, Göttingen, I, 165; HoEFER, F. (1842), Histoire de la chimie, I, 472; Ferguson, J. (1906), Bibliotheca Chemica, Glasgow, II, 591; THORNDIKE, L. (1946), V, 541; y PARTINGTON, J. R. (1961), II, 84-86.

52 PARACELSO (1584), Commentaria in archidoxorum libros $X$, Frankfurt.

53 PARACELSO, (1603), Operum medico-chimicorum sive Paradoxorum: Tomus Tertius Genuinus / Theophrasti Bombast, Ab Hohemheim, Dicti Paracelsi, Frankfurt.
} 
Teología en Padua y Venecia, y de quien Benedetto Croce dijera que en sus obras podemos ver una caricatura del Humanismo ${ }^{54}$.

Pero uno de los textos más leídos y conocidos en la Península durante los siglos XVI y XVII fue el de Conrad Gesner (1515-1565) Tesoro de los remedios secretos de Evónimo ${ }^{55}$. Desconocemos los motivos por los que Gesner decidió usar el pseudónimo de Evónimo Filiatro para titular su texto más famoso ${ }^{56}$, donde nos dio un gran número de quintas esencias, ya sean aguas, aceites, etc., preparadas tanto en el reino mineral, como en el animal y el vegetal. Sí sabemos que Gesner recopiló remedios secretos de Rupescissa, Arnau y Lulio, especialmente en su preparación química (es decir: por destilación o sublimación), también sabemos que Gesner dijo que fue Ulstad quien mejor expuso en qué consistía la quinta esencia, por lo que la serie de influencias está clara hasta el momento. pero quizá sea mejor verlo con sus propias palabras:

\begin{abstract}
«Llaman quinta esencia a una virtud o facultad soberana y celestial que posee cualquier planta, metal, animal, o parte de ellos [...] El primer autor en escribir sobre ella ha sido Raimundo Lulio. Hasta entonces era desconocida por todos los médicos y no se había escrito ni experimentado nada sobre ella. Luego comenzaron a ensayarla, entre otros, Juan de Rupescisa (que algunos piensan que es anterior a Ramón Llull, aunque yo creo que es contemporáneo), Jerónimo de Brunschwig, Felipe de Ulstad y algunos otros, no muchos, que escribieron alguna cosa sobre ella» ${ }^{57}$.
\end{abstract}

Pero este punto en común ha de situarse junto a otras características generales. En primer lugar, todos ellos tuvieron más textos donde no hablan de Alquimia ni de medicamentos químicos, que también fueron conocidos. En concreto Conrad Gesner, un magnífico observador de la Naturaleza, ya notó que la generación de los helechos se hacía a través de sus esporas en la obra Opera botanica ${ }^{58}$. En segundo lugar, todos estos autores, además de influir en Europa, como hemos visto, también lo fueron en la Península y de una forma muy concreta: hubieron de soportar el tamiz de la Inquisición. Gesner lo fue a través de lo concluido tras el $5^{\circ}$ Concilio de Florencia y lo dicho sobre la transustanciación; concretamente, la Inquisición de Toledo, en el año

54 Collona, F. (1724), Abregé de la doctrine de Paracelse et de ses archidoxes avec une explication de la nature des principes de la chyimie pour servir d'éclaircesssement aux traitez de cet auteur et des autres philosophes, París, d'Houry.

55 GESNER, C. (1555), Thesaurus evonimi philiatri de remediis secretis, liber physicus, medicus \& partim etiam chymicus, Lugduni; (1557), Thesaurus evonimi philiatri de remediis secretis, liber physicus, medicus \& partim etiam chymicus, Antonium Vicentum, Lugduni; (1559), Thesaurus evonimi philiatri de remediis secretis, liber physicus, medicus \& partim etiam chymicus, Tiguri, Andream Gesnerum.

56 THORNDIKE, L. (1946), V, 621.

57 GeSNER, C. (1996), Tesoro... (traducción del original latino, con introducción y notas de Andrés Manrique y Agustín Fernández), San Lorenzo de El Escorial, EDES, cap. XVII «La quinta esencia de los remedios», p. 281

58 GESNER, C. (1571), Opera botanica, Nüremberg. 
1593, hacía efectiva dicha censura ${ }^{59}$. Paracelso, ya lo fue en el Índice de Quiroga de 1583, a través de sus Chirurgias $^{60}$. Las censuras de textos de Paracelso en la Península son un tanto curiosas ${ }^{61}$, ya que, por ejemplo, sobre sus Pronósticos pareció haber otra vara de medir ${ }^{62}$. Sobre la censura a Lulio, las cosas fueron muy complicadas, especialmente desde la segunda mitad del siglo XVI hasta los primeros años del siglo XVII, e incluyeron largas disquisiciones entre los expertos, indecisiones y polémicas ${ }^{63}$.

\section{LOS TEXTOS PSEUDOLULIANOS}

Respecto de los textos de Alquimia atribuidos a Lulio, conocemos el auge de ediciones europeas durante todo el siglo XVI, especialmente en la segunda mitad del mismo. Estudios recientes, especialmente los de la profesora Michela Pereira han lanzado la idea de que el paracelsismo, y concretamente las nociones de Paracelso sobre la Medicina química, venían incluidas en las citadas ediciones alemanas de los textos pseudo-lulianos, aún más, parece que no hay duda sobre ello ${ }^{64}$. Al plantearnos la misma cuestión sobre lo ocurrido en la Península, hemos considerado esas mismas propuestas a partir de varios hechos. El primero es saber de qué textos y de qué ediciones nos podemos servir para poder corroborar esto. No obstante, sabemos que Paracelso citó a Lulio y a Vilanova más de treinta veces ${ }^{65}$.

En la Península contamos con varias de las ediciones de textos pseudolulianos que se hicieran en Alemania, especialmente las más importantes y conocidas hasta el año 1573. Hemos recogido todas las que comprenden los años que van desde la muerte de Paracelso, en el año 1541, hasta las primeras referencias escritas sobre él, a manos de Juan Fragoso (m.1597), médico y cirujano real, en el Antidotario que se incluye en su Chirurgia universal, editada por primera vez antes del año $1581^{66}$. Antes de analizar ligeramente estas ediciones, ya podemos hacer una comprobación

59 Censuras del epitome de Conrado Gesner, Archivo Histórico Nacional (A.H.N.), Inquisición., leg. 4436-15, 1593.

60 PARACElso (1573), Chirurgia Magna, Estrasburgo; Paracelso (1570), Chirurgia_Minor, Basilea.

61 Sobre la censura inquisitorial peninsular en cuanto a Ciencia se refiere: PARDO TOMÁs, J. (1991), Ciencia y censura. La Inquisición española y los libros científicos en los siglos XVI y XVII, Madrid, C.S.I.C.

62 Paracelso (s.f.), Prognosticatio, s.l.; Paracelso (1536), Prédiction du grand et sublime docteur Théophraste Paracelse, París.

63 PINTO CRESPO, V. (1982), «La censura inquisitorial, inquietud e incertidumbre: el caso Ramón Llull (1559-1610)», en Miscelánea de la U.A.M., Madrid, U.A.M., pp. 293-314.

64 PEREIRA, M. (1986), «Filosofia naturale lulliana e alchimia. Con l'inedito epilogo del 'Liber de secretis naturae seu de quinta essentia», en Rivista di Storia della Filosofia, 41, pp. 747-780.

65 LÓPEZ PIÑERO, J. M. (1994), p. 15, n. 14 sobre MUELLER, M. (1960), Registerband zu Sudhoffs Paracelsus Gesamt-Ausgabe. Medizinische, naturwissenschaftliche, pphilosophie Schriften, Eberle, p. 220.

66 Fragoso, J. (1581), Chirurgia uniuersal, Madrid, Vda. de Alonso Gómez. 
sobre la veracidad de la presencia de paracelsismo, incluso no solo a través de los textos lulianos, algo que veremos más adelante, sino, también, del concepto de quinta esencia que usó Fragoso, basado en sus lecturas de autores como Conrad Gesner y pseudo-Lulio, según sus propias palabras. Afortunadamente, la Historia de la Ciencia ya ha reparado en la influencia de Pseudo-Lulio, Rupescissa y Arnau en Paracelso en varias ocasiones ${ }^{67}$, incluso, como hiciera Walter Pagel, se han considerado fuentes de su obra ${ }^{68}$.

Las obras alquímicas pseudolulianas a las que me refiero son tres: El Testamentum, De secretis naturae y Codicilus. Respecto del primer texto, podemos consultar las dos primeras ediciones de los años 1566 y 1573 que hiciera en Colonia Ioannem Birckmann gracias a los varios ejemplares disponibles ${ }^{69}$. Es obligado plantearse la verdadera necesidad de la presencia de esta obra en la Península, y en su soporte impreso, ya que la existencia de manuscritos de fecha anterior es abundante ${ }^{70}$; aún más, cabe preguntarse quiénes leyeron estos textos, quiénes tuvieron capacidad para traerlos aquí y qué recepción causó entre sus lectores sobre sus ideas de la Alquimia y la Medicina química.

Sobre el De secretis naturae, existen, al menos, dos ediciones, siendo la primera especialmente curiosa ya que el título no concuerda con los de las demás ${ }^{71}$, más extenso y del mismo año, donde se incluye la no menos famosa carta al rey Roberto de Inglaterra, ya citada antes ${ }^{72}$. Es evidente que la intención de incluir en dicha carta, donde podemos ver que Lulio recogió las opiniones de Rupescissa, a esas alturas del siglo, es la de asentar y conectar la firmeza y categoría de los textos alquímicos atribuidos a Lulio con las nuevas ediciones hechas a manos de los paracelsistas alemanes más afamados ${ }^{73}$, los protagonistas del paracelsian revival que hablara Lynn

67 LÓPEZ PiÑERO, J. M. (1994), y MonTIEL, L. (1998), «La introducción del remedio mineral», en Historia del medicamento, II, pp. 123-144.

68 LÓPeZ PiÑERO, J. M. (1994), p. 15, n. 14; sobre PAGel, W. (1958), Paracelsus. An Introduction to Philosophical Medicine in the Era of the Renaissance, Basel, p. 241 y ss.

69 PSEUDO-LULIO, R. (1566), Raymundi Lullii testamentum, duobus libris universam artem chymicam complectens, antehaenunquam excusum / item eiusdem compendium animae tranmutationis artis metallorum, absolutem iam \& perfectum, Colonia; Pseudo-Lulio, (1573), Testamentum Raymundi Lulii..duobus libris universam artem chymicam complectens; item eiusdem compendium animae transmutationis artis metallorum, secunda aeditio, multorum exemplarium collatione infinitus locis costigatior, Colonia.

70 Ejemplares en: Barcelona, Biblioteca de Catalunya, 1728; Madrid, Biblioteca Nacional, ms. 17721 y ms. 18431; Palma de Mallorca, Colegio Pontificio de Nra. Sra. de la Paciencia, leg. 7 y Palma de Mallorca, Biblioteca del Convento de S. Francisco, 16 (2/32) y 17 (3/9).

71 Pseudo-Lulio, R. (1567a), De secretis naturos, Colonia.

72 Pseudo-Lulio, R. (1567b), Raymundi Lullii de secretis naturae, seu de quinta essentia liber unus, in tres distinctiones divisus, omnibus iam partibus absolutus / adiecta est eiusdem apistola ad regem Robertum de accurtatione lapidis philosophorum: eius adiuntus est tractatus de aqua ex scriptis Raymundi super accurtationis epistolam ab artis studioso collectus, Colonia.

73 Internet: http://www.nlm.gov/exhibition/paracelsus. «The medical chemistry of the paracelsians». 


\section{LA INFLUENCIA DE LA ALQUIMIA MEDIEVAL HISPANA EN LA EUROPA MODERNA}

Thorndike $\mathrm{f}^{74}$. Y la mejor forma era incluir en las nuevas ediciones textos a conocidos, como la citada carta, que ya fuera incluida también en la Margarita de Janus Lacinius ${ }^{75}$, aunque con un título más completo que en el ejemplar de Palma de Mallor$\mathrm{ca}^{76}$. Por cierto que Lacinius dijo de Lulio que

«...praeteribus temporibus plures libris secretos in arte transmutationem composuissem \& librum omnium secretissimum de lapidibus pretiosis secundum virtutes suas compositis» ${ }^{77}$.

Respecto de Codicilus, o Vade mecum, contamos con la segunda edición de Birckmann, del año $1572^{78}$, la edición del mismo año de Petrus Pernam ${ }^{79}$ y con otra, muy curiosa aparecida dos años después, ya que confirma nuestra tesis, cuyo director fue el paracelsista Miguel Toixites (1515-1581), con el título Raimundi Lullii...libelli aliquot chemici nunc primum, excepto vade mecum, in lucem opera doctore Toxitae editi ${ }^{80}$.

No son la únicas ediciones alemanas que se pudieron leer en la Península durante el siglo XVI. También tenemos el texto Compendium artis alchemiae et naturalis philosophiae, incluido en la compilación Auriferae Artis de Conrad Waldrick ${ }^{81}$. Se trata de una breve práctica alquímica, donde se cita el Testamentum, que trata de la extracción de la quinta esencia, aunque es un proceso visto como mágico. Dicha obra sólo puede ser consultada como manuscrito en el ejemplar existente en la Biblioteca de Catalunya ${ }^{82}$, a pesar que hay otras ediciones europeas en solitario, como la de Nüremberg de 1547 y la de Colonia de 1567, según nos dijeran Roger \& Durán ${ }^{83}$. Si bien no es apreciable en este texto las influencias de los paracelsianos, sí es nítida, en cambio, la de los textos de Rupescissa, por lo que hemos de analizar algo más lo ocurrido con el Auriferae Artis.

74 THORNDIKE, L. (1946), V, 617-651.

75 LACINIUS, J. (1546), Pretiota Margarita Novella de Thesauro, ac pretiosissimo lapide; Collectanea ex Arnaldo, Raymondo (sic), Rashi..., Aldi, Venecia.

76 PSEUdO-LULIO, R. (1546), «Epistola sive epitome Raymundi Lulli», en Lacinius, J. (1546), p. 160-161.

77 LACINIUS, J. (1546), p. 160.

78 PSEUdO-Lulio, R. (1542), Codicilus seu de vade mecum Raymundi Lulli in quo fontes alchimiae artis ac philosophiae reconditioria uberrime traduntur, secunda editio, Haeredes Arnoldi Birckmanni, Colonia.

79 PSEUdO-LULIO, R. (1572), Raimundi Lulii...libeli aliquot chemici nunc primum excepto vade mecum... editi, continens testamentum novisimum integrum, elucidatio vocabularium eius, lux mercuriorum, experimenta, vade mecum, compendium chimiae transmutationis artis, epistola accurtationis lapidis metallorum, de medicinis secretissimus, lignum vitae, Petrus Pernam.

80 (1574), Conradi Waldkirchi, Basilea. 1574.

81 Edición manejada: Biblioteca del Real Monasterio de San Lorenzo de El Escorial, 109-VII-32, parte III, p. 83.

82 Signatura: 1740

83 Rogent, E. DuRÁN, E. (1927), Bibliografía de les impresions lul.lianes, Barcelona, pp. 94 y 109. 
Uno de los primeros especialistas en editar colecciones de textos alquímicos fue Guillermo Gratarolo, personaje estudiado por Lynn Thorndike ${ }^{84}$ y por Günter Jütnner en su tesis doctoral ${ }^{85}$. En vez de publicar muchos textos alquímicos aislados, que también hiciera, los recogió y editó, aumentando considerablemente el número de los contenidos en colecciones anteriores. Así, su Verae Alchemiae artisque metallicae, del año 1561, contiene cincuenta y tres tratados ${ }^{86}$. Once años después, en 1572, apareció su Alchemiae quam vocant, con un contenido que corresponde, casi en su integridad, al Verae Alchemiae ${ }^{87}$, y no siendo, como dice Ferguson, una reimpresión ${ }^{88}$, ya que en su tomo II, páginas 359-485, se incluye el De quinta essentia de Juan de Rupescissa, obra que Gratarolo mandó imprimir separadamente en 1561. En el mismo año de 1572, su impresor Petrus Pernam sacó a la luz una nueva compilación por cuenta propia (se ignora el papel de Gratarolo en esta edición), bajo el título de Auriferae Artis $^{89}$, republicado en 1593, aunque ya no como impresor, labor que recayó en Conrad Waldrick ${ }^{90}$ y considerablemente aumentado en 1610, esta vez con el título de Artis Auriferae, y en tres volúmenes y cuarenta y seis textos ${ }^{91}$.

A modo de conclusión, perece evidente que las ideas de Paracelso están presentes en la Península desde principios de los años setenta del siglo XVII, con seguridad, y con alguna duda, desde los años sesenta de dicho siglo. También que la Alquimia y los alquimistas medievales peninsulares inspiraron en gran medida a los protagonistas de los momentos iniciales de la Medicina química europea. Así mismo, las ideas de dichas personas (Lulio, Arnau y Rupescissa) salieron, entraron y fueron conocidas en dos aspectos: tal y como habían vuelto desde Europa y tal y como se desarrollaron

84 THORNDIKE, L. (1946), V, pp. 600-616.

85 JÜTNNER, G. (1969), Wilheim Gratarolus. Benedikt Aretius. Naturwissenschaftliche Beziehungen der Universität Marburg zur Schweiz im 16 Jahrhundert, Marburgo, Servicio de Publicaciones de Tesis Doctorales de la Universidad de Marburgo.

86 (1651), Verae Alchemiae Artisque Metallicae, citra aenigmata, Doctrina, certusque modus, scriptis tum novis tum ueteribus nunc primum et fideliter maiori ex parte eitis, compehensus: quorum elenchum a Praefatione reperies. Habes, amice, et ueluti sepulta iacuerunt: quorum editionis rationem im Paefatione ad philosophos Chemistas paucis intelliges, Basilea, Henric Petri et Petrus Pernam.

87 (1572), Alchemiae, quam vocant, artisque metallicae, doctrina, certusque modus, scriptis tum nouis, tum veteribus, duobus his voluminus comprehensus. Quorum Elenchum a prafectione reperies, Basilea, Petrus Pernam.

88 FERguSON, J. (1906), I, p. 342

89 (1572), Auriferae Artis, quam Chemiam vocant, antiquissimi Authores, sive Turba Philosophorum, Basilea, Petrus Pernam.

90 Pernam, P. (1593), Auriferae Artis, Conrad Waldrick, Basilea. Waldrick se especializó en la edición de textos alquímicos hasta se muerte, en 1613.

91 Pernam, P. (1610), Artis Auriferae, quam Chemiam vocant, volumina duo, quae continent Turbam Philosophorum, aliosque antiquiss. Auctores, quae versa pagina indicat. Accessit noviter volumen tertium... Omnia hactenus nunquam visa nec edita, Cum Indicibus rerum et verborum locupletiss, Basilea, C. Waldrick. 
en el interior. Estas dos facetas, dado que hubo manuscritos e impresos de dichos autores de ambas y en los mismos años, pudieron, y seguramente así ocurrió, entrar en contacto. Este contacto resulta ser de suma importancia para el estudio de la Medicina química peninsular ya que estamos hablando de las palabras de los mismos autores, pero vistas desde la Alquimia y desde la Medicina química. El efecto que generó dicho contacto, visto a través de las opiniones vertidas en las obras de las figuras de la Sanidad y la Terapéutica peninsulares, es un componente fundamental para el estudio de la iatroquímica de fines del siglo XVI y principios del siglo XVII. 
